\title{
Second Generation Antipsychotics Improve Sexual Dysfunction in Schizophrenia: A Randomised Controlled Trial
}

\author{
Ahmed Mahmoud, ${ }^{1}$ Karen P. Hayhurst, ${ }^{2}$ Richard J. Drake, ${ }^{1}$ and Shôn W. Lewis ${ }^{1}$ \\ ${ }^{1}$ Mental Health and Neurodegeneration Research Group, The University of Manchester, Manchester M13 9PL, UK \\ ${ }^{2}$ National Drug Evidence Centre (NDEC), School of Community Based Medicine, The University of Manchester, \\ Manchester M15 6SZ, UK
}

Correspondence should be addressed to Karen P. Hayhurst, karen.hayhurst@manchester.ac.uk

Received 5 November 2009; Revised 24 August 2010; Accepted 4 November 2010

Academic Editor: D. Naber

Copyright ( 2011 Ahmed Mahmoud et al. This is an open access article distributed under the Creative Commons Attribution License, which permits unrestricted use, distribution, and reproduction in any medium, provided the original work is properly cited.

The impact of antipsychotic drug treatment on sexual function was investigated during a randomised trial comparing first generation antipsychotics (FGAs) to (nonclozapine) second generation antipsychotics (SGAs). Sexual function and quality of life were (rater-blind) assessed in 42 patients with DSM-IV schizophrenia (aged 18-65) using the self-report version of the Derogatis Interview for Sexual Function (DISF-SR) and the Heinrichs Quality of Life Scale (QLS), prior to, and 12 weeks following, a change in medication from an FGA drug to either an FGA or SGA drug. SGAs significantly improved sexual function compared to FGAs. Change in sexual function was associated with change in quality of life. Where impaired sexual functioning is a distressing adverse effect of treatment with an FGA agent, consideration should be given to switching to an SGA.

\section{Introduction}

Sexual dysfunction is common in people with schizophrenia [1] affecting around a half of all patients [2-4] and likely to contribute to impaired quality of life [5-7]. Little systematic research has been carried out to date on the sexual behaviour of schizophrenic patients and a Cochrane review recently called for further research on the management of antipsychotic treatment-related sexual dysfunction [8].

Patients themselves report that the sexual side effects of medication are distressing [9]. For example, Finn and colleagues [10] found that patients with schizophrenia rated impotence, as a treatment side effect, as worse than any symptoms of the illness itself. Men may perceive sexual dysfunction as more distressing than women [11], but the experience of sexual dysfunction in women with schizophrenia has been underresearched. Sexual dysfunction can be a significant reason for nonadherence to prescribed antipsychotic treatment $[1,2,12-15]$.

The relationship between antipsychotic drugs and sexual dysfunction is mediated in part by antipsychotic blockade of pituitary D2 receptors increasing prolactin secretion, although direct correlations have not been established between raised prolactin levels and clinical symptoms [16-18]. More recent studies highlight that a variety of mechanisms are likely to contribute to antipsychotic-related sexual dysfunction, including hyperprolactinaemia, sedation, and antagonism of a number of neurotransmitter receptors $(\alpha$-adrenergic, dopaminergic, histaminic, and muscarinic) $[19,20]$.

Conventional, or first generation antipsychotic (FGA) drugs are associated with sexual side effects. Ghadirian and colleagues [21] found that up to $60 \%$ of men and $33 \%$ of women on FGAs reported changes in the quality of orgasm. A further study reported that chlorpromazine was associated with problems of orgasm and sulpiride with problems of arousal in female patients [3]. A Cochrane review highlights increased prolactin levels seen with sulpiride treatment [22]. Recent research demonstrates that as sulpiride is less lipophilic than other antipsychotics it has relatively high concentrations in the pituitary (outside the blood-brain barrier) compared to brain parenchyma [23]. This means that hyperprolactinaemia is more likely to occur at clinical doses of sulpiride. 
Individual second generation antipsychotic (SGA) drugs appear to be associated with less sexual dysfunction than that attributable to FGAs. One recent review concluded that risperidone had the largest impact on sexual dysfunction, followed by FGA drugs, then olanzapine, then clozapine, then quetiapine and, finally, aripiprazole [19]. Antipsychotic drugs with high D2-binding affinity have been identified as more likely to cause sexual dysfunction $[1,24]$ and risperidone may be associated with higher prolactin elevation than other SGAs [25-27]. Amisulpride is less lipophilic than risperidone or other SGAs and this may (as for sulpiride) explain why it is particularly identified with hyperprolactinaemia [26]. Bearing in mind the limitations associated with its design, data from the SOHO study, a large observational study funded by Lilly, indicates an advantage to both olanzapine and quetiapine, compared with risperidone and FGA drugs, across areas of sexual dysfunction [28-30]. A previous European study concluded that there were no real benefits of any of the SGAs over FGA treatment, apart from that seen with quetiapine in short-term trials [31]. Further studies have confirmed the association of both quetiapine $[1,32,33]$ and aripiprazole $[34,35]$ with less severe sexual dysfunction.

Sexual dysfunction is a difficult area to investigate. Sexual problems may be underreported to clinicians who, in turn, may underestimate the sexual side effects of antipsychotic treatment $[1,2,6,7,13,29,31]$. Researchers in the field have used a variety of assessment tools to measure sexual function, leading to difficulty when reviewing the published literature.

We set out to examine sexual dysfunction in a subgroup of participants in a large trial. Patients already taking, but unresponsive to, an FGA were randomised to change to either another FGA or a non-clozapine SGA for 12 weeks while sexual function and quality of life were assessed.

We tested two hypotheses. Firstly, that sexual dysfunction contributes measurably to reduced health-related quality of life in patients with schizophrenia. Secondly, that sexual function and hence quality of life is better in patients with schizophrenia on SGAs than FGAs.

\section{Materials and Methods}

Patients $(N=42)$ were treated with an FGA drug prior to randomisation into the study. The study was conducted alongside CUtLASS (Cost Utility of the Latest Antipsychotics in Severe Schizophrenia [36-38]: a multicentre RCT that compared FGAs with SGA drugs. All participants of the substudy were recruited from one centre. The North West MultiCentre Research Ethics Committee granted ethical approval with local approval obtained from each participating district.

2.1. Inclusion Criteria. Patients had a diagnosis of DSM-IV schizophrenia (and related disorders). The sample comprised patients recruited to CUtLASS $(N=20)$ and additional patients recruited from clinical practice $(N=22)$. All participants entered the study because of an identified need for a change to their FGA treatment, either because of ineffectiveness or intolerable side effects. No participants were referred because of sexual side effects. Patients were aged 18 to 65 years of both sexes. Patients receiving anticholinergic drugs were included.

2.2. Exclusion Criteria. Excluded patients included those with a diagnosis of schizoaffective disorder, patients with a comorbid history of alcohol or drug misuse within the last 12 months, and patients receiving treatment with more than one antipsychotic drug at baseline.

2.3. Randomisation. Randomisation, of all included patients, took place via a remote telephone service provided by the Medical Statistics Department at the Paterson Institute, Christie Hospital, Manchester, UK. The method of allocation was permuted blocks within strata with block sizes varying at random between four and 12 . Arrangements were in place to ensure that the patient commenced their new antipsychotic drug treatment within hours of randomisation to either an FGA or an SGA drug. All subjects had a change to their antipsychotic drug and all completed the 12 -week period on their assigned treatment. Randomised treatment allocation was revealed to the responsible clinician, the CUtLASS trial manager and the participant's GP.

2.4. Measures. Primary and secondary outcome measures used, respectively, were the self-report Derogatis Interview for Sexual Function (DISF-SR) [39] and the (rater-blinded) Quality of Life Scale (QLS) [40]. The Derogatis interview is a widely used, brief, self report, multidimensional and gender-keyed instrument designed to measure quality of current sexual functioning across five primary domains: sexual cognition and fantasy (5 items); sexual arousal (5 items); sexual behaviour and experiences (5 items); orgasm (6 items); sexual drive and relationship (4 items). The first three of these key domains are scored using a nine-point scale (from "0" as "not at all" through to " 8 " as " 4 or more times per day"); orgasm is scored using a five-point scale (from " 0 " as "not at all" to " 4 " as "extremely"); the fifth domain, sexual drive, and relationship, is scored using a combination of nine- and five-point scales. The aggregate total score can be used repeatedly throughout efficacy or effectiveness studies without any significant practice effects or loss of validity [39].

The QLS [40] is the most widely used quality of life scale in the evaluation of psychopharmacological treatments for schizophrenia [41]. The 21-item scale covers four domains: social relationships; instrumental role functioning; intrapsychic foundations; activities of daily life. The QLS has been shown to be both sensitive to change and of clinical relevance [42].

2.5. Statistical Analysis and Power. Results were analysed using SPSS version 15 to carry out analyses of covariance (ANCOVAs) and correlational analyses (Pearson's $r$ and Spearman's rho). An a priori power calculation suggested that the sample size obtained would have $90 \%$ power to detect a difference of 30 points on the DISF-SR between the two treatment groups, which was selected as a difference 
TABle 1: Demographic Characteristics.

\begin{tabular}{|c|c|c|c|c|}
\hline & FGA $\operatorname{Arm}(N=22)$ & $\operatorname{SGA} \operatorname{Arm}(N=20)$ & $P$ & test \\
\hline \multicolumn{5}{|l|}{ Age } \\
\hline Mean/SD & $38.4(10.0)$ & $43.0(11.7)$ & .173 & $t$ test \\
\hline Median/Range & $39.6 / 18-53$ & $45.9 / 19-57$ & & \\
\hline \multicolumn{5}{|l|}{ Illness duration } \\
\hline Mean/SD & $14.4(8.7)$ & $18.6(11.9)$ & & \\
\hline Median/Range & $13.0 / 0.5-34$ & $24.6 / 1-33$ & .182 & Mann-Whitney \\
\hline \multicolumn{5}{|l|}{ Gender } \\
\hline Male (\%) & $19(86 \%)$ & $12(60 \%)$ & .052 & Chi Square \\
\hline \multicolumn{5}{|l|}{ Ethnicity } \\
\hline White (\%) & $20(91 \%)$ & $18(90 \%)$ & 1.000 & Chi Square \\
\hline
\end{tabular}

TABLE 2: This table shows antipsychotic drug treatment received by the two groups.

\begin{tabular}{lccccc}
\hline & FGA Arm $(N=22)$ & & SGA Arm $(N=20)$ & Dose range \\
$N$ & Drug & Dose range & $N$ & Drug & $400 \mathrm{mg}$ \\
\hline 2 & Flupentixol & $3-6 \mathrm{mg}$ & 2 & Amisulpride & $5-15 \mathrm{mg}$ \\
1 & Haloperidol & $3 \mathrm{mg}$ & 7 & Olanzapine & Quetiapine \\
3 & Sulpiride & $400-2000 \mathrm{mg}$ & 7 & Risperidone & $3-4 \mathrm{mg}$ \\
1 & Trifluoperazine & $15 \mathrm{mg}$ & 4 & & \\
7 & Zuclopenthixol & $4-20 \mathrm{mg}$ & & \\
1 & Flupentixol depot & $402 / 52$ & & \\
1 & Fluphenazine depot & $252 / 52$ & & \\
1 & Pipotiazine palm. & $503 / 52$ & & \\
5 & Zuclopenthixol depot & $2004 / 52-4002 / 52$ & & & \\
\hline
\end{tabular}

that both appeared clinically relevant and related to previous findings of differences between treatment groups [43].

\section{Results}

Table 1 shows demographic characteristics of the two treatment groups. Thirty-one men and 11 women with a mean age of 41 years (range 18 to 57 years) were randomised.

The two groups were statistically comparable in terms of their ethnicity and age. The FGA arm had a lower median duration of illness and a higher proportion of males, compared with the SGA arm, although neither measure differed significantly between the two groups.

Table 2 shows antipsychotic drug treatment received by the two groups.

Table 3 shows sexual function scores of the two treatment groups over the two assessment time points.

Mean baseline total and subsection scores on the DISFSR differed between the two groups (Table 3), but not significantly. Table 3 also shows that mean DISF-SR scores of the FGA-treated group worsened somewhat between the two time points.
There was no significant correlation between DISF-SR total score and QLS total score at baseline $(r=0.167, P=$ $.290)$ and a weak (nonsignificant) correlation at week $12(r=$ $0.293, P=.063$ ). The trend relationship seen by week 12 of the study resulted from a significant correlation between score on QLS subscale 1 (interpersonal relations \& social network) and score on Section 3 of the DISF-SR (sexual behaviour \& experience: Spearman's rho $=0.356, P=.022$ ). There was a significant correlation between change in DISFSR total score and change in QLS total score from baseline to week 12 (Spearman's rho $=0.627, P<.001$ ).

Twelve week DISF-SR total was modelled with an analysis of covariance. Final sexual function was significantly better $(F=7.45, P=.010)$ for patients randomised to an SGA $(N=20)$, compared to patients receiving an FGA $(N=22)$, covarying for baseline score and gender. Adjusted 12-week marginal mean DISF-SR score was 58.0 in the SGA group and 37.7 in the FGA group (difference 20.3, 95\% confidence interval 5.2, 35.4). Age was dropped as a covariate as it was not independently significant. There was no significant difference between groups in final QLS total $(F=0.421, P=$ 
TABLE 3: DISF-SR score.

\begin{tabular}{|c|c|c|c|c|c|}
\hline \multirow[t]{2}{*}{ DISF-SR Mean (SD) } & \multicolumn{2}{|c|}{ FGA Group $(N=22)$} & \multicolumn{2}{|c|}{ SGA Group $(N=20)$} & \multirow[b]{2}{*}{ Baseline comparison } \\
\hline & Baseline & Week 12 & Baseline & Week 12 & \\
\hline Section 1: sexual cognition/fantasy & $15.5(12.7)$ & $13.8(10.6)$ & $10.4(9.3)$ & $16.2(11.0)$ & $P=.130^{\dagger}$ \\
\hline Section 2: sexual arousal & $10.2(8.2)$ & $9.3(8.9)$ & $7.0(8.4)$ & $9.5(9.0)$ & $P=.202^{\dagger}$ \\
\hline Section 3: sexual behaviour/experience & $6.7(5.8)$ & $6.4(5.9)$ & $4.6(6.8)$ & $6.8(8.5)$ & $P=.085^{\ddagger}$ \\
\hline Section 4: orgasm & $9.1(7.2)$ & $8.0(6.8)$ & $5.6(8.0)$ & $9.0(8.0)$ & $P=.128^{\dagger}$ \\
\hline Section 5: sexual drive & $10.6(4.5)$ & $9.6(5.0)$ & $9.0(4.6)$ & $11.1(5.0)$ & $P=.236^{\dagger}$ \\
\hline Total Score & $52.6(32.7)$ & $47.5(32.3)$ & $36.4(33.7)$ & $52.6(37.3)$ & $P=.121^{\dagger}$ \\
\hline
\end{tabular}

t $t$-test.

${ }^{\ddagger}$ Mann-Whitney.

NB: A higher score on the DISF-SR scale is indicative of better sexual functioning.

.521) or QLS subscales, covarying for gender and baseline score.

\section{Discussion}

Regarding the first hypothesis, sexual dysfunction did not have a straightforward relationship with reduced quality of life. It was unrelated at baseline but improvement in sexual function had a weak to moderate relationship with improving quality of life. This drove a trend-level association at final assessment. It may be that changes in sexual function have a more direct effect on quality of life than static sexual function, perhaps because over time there is a degree of adjustment to dysfunction. It is also worth noting that the QLS is an objective measure of quality of life, influenced by social function as well as participants' subjective experience; subjective quality of life may be more strongly related.

Previous studies have linked male sexual dysfunction with poor quality of life in schizophrenia [6]. Although our results do not confirm this, again, the QLS may not be the best type of measure to detect a relationship. Total score on the QLS may not have sufficient sensitivity to detect sexual functioning-related changes in quality of life, and there is an argument for examining subscales of the QLS and DISF-SR.

In terms of the second of our hypotheses, those who were in the SGA group had significantly better sexual function by the end of the trial. This suggests that switching from an FGA to an SGA can improve sexual function significantly. Note that none of the participants were referred into the study because of the sexual side effects of antipsychotic drug treatment. Sexual side effects can lessen with time following a treatment switch [44]. This reduction over time could, in part, explain the improvement in sexual function seen in the SGA group but fails to account for the deterioration seen in the FGA group.

The study had a number of limitations. As stated earlier, the nature of the QLS, being determined largely by the deficit symptoms of schizophrenia [45], may explain the lack of effect of drug type on measured quality of life. Other treatment-related side effects, such as parkinsonism or sedation, which may also affect sexual function, were not measured in the study. The relatively small sample size did not permit an analysis of individual drugs. At the time of the study, some SGA drugs, such as ziprasidone and paliperidone, were not available in the UK, whilst others, such as zotepine, were little used. Female patients made up a relatively small proportion of those recruited limiting the generalisability of the findings. The study did, however, have the advantage of being a prospective, randomised comparison, one of a few to assess sexual function comprehensively. The self-report Derogatis scale was selected for this study because of its high validity and reliability.

These findings add to the evidence that SGA drugs, as a group, have less adverse sexual side effects than those associated with FGA treatment. As an RCT of a change in medication it confirms the implication of existing nonrandomised survey data that switching antipsychotics can reduce the risk of treatment-associated sexual dysfunction $[12,19,25]$. Of course, it is not clear what effect switching within SGAs or from SGA to FGA would be, so it is uncertain whether this is an effect of switching class.

\section{Conclusions}

Switching from FGAs to SGAs improved sexual function compared to switching within FGAs. The relationship between the self-report measure of sexual function and the assessor-rated, objective quality of life measure used was complex, but it appeared that change in sexual function was associated with change in quality of life. Where impaired sexual functioning is a distressing adverse effect of treatment with an FGA agent consideration should be given to switching to an SGA.

\section{Acknowledgments}

This study was conducted alongside the CUtLASS trials (Cost utility of the latest antipsychotics in severe schizophrenia, [36-38]) which were funded by NHS R\&D Health Technology Assessment. The authors are grateful to reviewers for their comments on an earlier draft of this paper. 


\section{References}

[1] D. L. Kelly and R. R. Conley, "A randomized double-blind 12 -week study of quetiapine, risperidone or fluphenazine on sexual functioning in people with schizophrenia," Psychoneuroendocrinology, vol. 31, no. 3, pp. 340-346, 2006.

[2] M. Dossenbach, A. Hodge, M. Anders et al., "Prevalence of sexual dysfunction in patients with schizophrenia: international variation and underestimation," International Journal of Neuropsychopharmacology, vol. 8, no. 2, pp. 195-201, 2005.

[3] S. M. Smith, V. O'Keane, and R. Murray, "Sexual dysfunction in patients taking conventional antipsychotic medication," British Journal of Psychiatry, vol. 181, pp. 49-55, 2002.

[4] A. Üçok, C. İncesu, T. Aker, and Ş. Erkoç, "Sexual dysfunction in patients with schizophrenia on antipsychotic medication," European Psychiatry, vol. 22, no. 5, pp. 328-333, 2007.

[5] P. Assalian, R. Fraser, R. Tempier, and D. Cohen, "Sexuality and quality of life of patients with schizophrenia," International Journal of Psychiatry in Clinical Practice, vol. 4, no. 1, pp. 29-33, 2000.

[6] M. Olfson, T. Uttaro, W. H. Carson, and E. Tafesse, "Male sexual dysfunction and quality of life in schizophrenia," Journal of Clinical Psychiatry, vol. 66, no. 3, pp. 331-338, 2005.

[7] J. Peuskens, P. Sienaert, and M. De Hert, "Sexual dysfunction: the unspoken side effect of antipsychotics," European Psychiatry, vol. 13, supplement 1, pp. 23s-30s, 1998.

[8] M. M. Berner, M. Hagen, and L. Kriston, "Management of sexual dysfunction due to antipsychotic drug therapy," Cochrane Database of Systematic Reviews, no. 1, Article ID CD003546, 2007.

[9] M. Lambert, P. Conus, P. Eide et al., "Impact of present and past antipsychotic side effects on attitude toward typical antipsychotic treatment and adherence," European Psychiatry, vol. 19, no. 7, pp. 415-422, 2004.

[10] S. E. Finn, J. M. Bailey, R. T. Schultz, and R. Faber, "Subjective utility ratings of neuroleptics in treating schizophrenia," Psychological Medicine, vol. 20, no. 4, pp. 843-848, 1990.

[11] W. K. H. Fakhoury, D. Wright, and M. Wallace, "Prevalence and extent of distress of adverse effects of antipsychotics among callers to a United Kingdom national mental health helpline," International Clinical Psychopharmacology, vol. 16, no. 3, pp. 153-162, 2001.

[12] A. J. Cutler, "Sexual dysfunction and antipsychotic treatment," Psychoneuroendocrinology, vol. 28, supplement 1, pp. 69-82, 2003.

[13] D. L. Kelly and R. R. Conley, "Sexuality and schizophrenia: a review," Schizophrenia Bulletin, vol. 30, no. 4, pp. 767-779, 2004.

[14] D. O. Perkins, "Predictors of noncompliance in patients with schizophrenia," Journal of Clinical Psychiatry, vol. 63, no. 12, pp. 1121-1128, 2002.

[15] S. Smith, "Effects of antipsychotics on sexual and endocrine function in women: implications for clinical practice," Journal of Clinical Psychopharmacology, vol. 23, no. 3, pp. S27-S32, 2003.

[16] M. Hamner, "The effects of atypical antipsychotics on serum prolactin levels," Annals of Clinical Psychiatry, vol. 14, no. 3, pp. 163-173, 2002.

[17] H. Knegtering, R. van den Bosch, S. Castelein, R. Bruggeman,
S. Sytema, and J. van Os, "Are sexual side effects of prolactinraising antipsychotics reducible to serum prolactin?" Psychoneuroendocrinology, vol. 33, no. 6, pp. 711-717, 2008.

[18] P. Malik, "Sexual dysfunction in schizophrenia," Current Opinion in Psychiatry, vol. 20, no. 2, pp. 138-142, 2007.

[19] M. Baggaley, "Sexual dysfunction in schizophrenia: focus on recent evidence," Human Psychopharmacology, vol. 23, no. 3, pp. 201-209, 2008.

[20] P. M. Haddad and S. G. Sharma, "Adverse effects of atypical antipsychotics: differential risk and clinical implications," CNS Drugs, vol. 21, no. 11, pp. 911-936, 2007.

[21] A. M. Ghadirian, G. Chouinard, and L. Annable, "Sexual dysfunction and plasma prolactin levels in neuroleptic-treated schizophrenic outpatients," Journal of Nervous and Mental Disease, vol. 170, no. 8, pp. 463-467, 1982.

[22] B. G. Soares, M. Fenton, and P. Chue, "Sulpiride for schizophrenia," Cochrane Database of Systematic Reviews, no. 1, Article ID CD001162, 2000.

[23] R. Arakawa, M. Okumura, H. Ito et al., "Positron emission tomography measurement of dopamine $\mathrm{D}_{2}$ receptor occupancy in the pituitary and cerebral cortex: relation to antipsychotic-induced hyperprolactinemia," Journal of Clinical Psychiatry, vol. 71, no. 9, pp. 1131-1137, 2010.

[24] P. M. Haddad and A. Wieck, "Antipsychotic-induced hyperprolactinaemia: mechanisms, clinical features and management," Drugs, vol. 64, no. 20, pp. 2291-2314, 2004.

[25] C. Bushe, D. Yeomans, T. Floyd, and S. M. Smith, "Categorical prevalence and severity of hyperprolactinaemia in two UK cohorts of patients with severe mental illness during treatment with antipsychotics," Journal of Psychopharmacology, vol. 22, supplement 2, pp. 56-62, 2008.

[26] P. Falkai, T. Wobrock, J. Lieberman et al., "World Federation of Societies of Biological Psychiatry (WFSBP) guidelines for biological treatment of schizophrenia-part 1: acute treatment of schizophrenia," World Journal of Biological Psychiatry, vol. 6, no. 3, pp. 132-191, 2005.

[27] M. Hummer and J. Huber, "Hyperprolactinaemia and antipsychotic therapy in schizophrenia," Current Medical Research and Opinion, vol. 20, no. 2, pp. 189-197, 2004.

[28] I. Bitter, B. R. Basson, and M. R. Dossenbach, "Antipsychotic treatment and sexual functioning in first-time neuroleptictreated schizophrenic patients," International Clinical Psychopharmacology, vol. 20, no. 1, pp. 19-21, 2005.

[29] M. Dossenbach, Y. Dyachkova, S. Pirildar et al., "Effects of atypical and typical antipsychotic treatments on sexual function in patients with schizophrenia: 12-month results from the Intercontinental Schizophrenia Outpatient Health Outcomes (IC-SOHO) study," European Psychiatry, vol. 21, no. 4, pp. 251-258, 2006.

[30] M. Lambert, J. M. Haro, D. Novick et al., "Olanzapine vs. other antipsychotics in actual out-patient settings: six months tolerability results from the European schizophrenia out-patient health outcomes study," Acta Psychiatrica Scandinavica, vol. 111, no. 3, pp. 232-243, 2005.

[31] J. Bobes, M. P. García-Portilla, J. Rejas et al., "Frequency of sexual dysfunction and other reproductive side-effects in patients with schizophrenia treated with risperidone, olanzapine, quetiapine, or haloperidol: the results of the EIRE study," Journal of Sex and Marital Therapy, vol. 29, no. 2, pp. 125-147, 2003.

[32] M. J. Byerly, P. A. Nakonezny, B. M. Bettcher, T. Carmody, 
R. Fisher, and A. J. Rush, "Sexual dysfunction associated with second-generation antipsychotics in outpatients with schizophrenia or schizoaffective disorder: an empirical evaluation of olanzapine, risperidone, and quetiapine," Schizophrenia Research, vol. 86, no. 1-3, pp. 244-250, 2006.

[33] R. Knegtering, S. Castelein, H. Bous et al., "A randomized open-label study of the impact of quetiapine versus risperidone on sexual functioning," Journal of Clinical Psychopharmacology, vol. 24, no. 1, pp. 56-61, 2004.

[34] L. Hanssens, G. L'Italien, J. Y. Loze, R. N. Marcus, M. Pans, and W. Kerselaers, "The effect of antipsychotic medication on sexual function and serum prolactin levels in communitytreated schizophrenic patients: results from the Schizophrenia Trial of Aripiprazole (STAR) study (NCT00237913)," BMC Psychiatry, vol. 8, article no. 95, 2008.

[35] A. Mir, K. Shivakumar, R. J. Williamson, V. McAllister, V. O'Keane, and K. J. Aitchison, "Change in sexual dysfunction with aripiprazole: a switching or add-on study," Journal of Psychopharmacology, vol. 22, no. 3, pp. 244-253, 2008.

[36] P. B. Jones, T. R. E. Barnes, L. Davies et al., "Randomized controlled trial of the effect on quality of life of secondvs first-generation antipsychotic drugs in schizophrenia: cost utility of the latest antipsychotic drugs in schizophrenia study (CUtLASS 1)," Archives of General Psychiatry, vol. 63, no. 10, pp. 1079-1087, 2006.

[37] S. W. Lewis, T. R. E. Barnes, L. Davies et al., "Randomized controlled trial of effect of prescription of clozapine versus other second-generation antipsychotic drugs in resistant schizophrenia," Schizophrenia Bulletin, vol. 32, no. 4, pp. 715723, 2006.

[38] S. W. Lewis, L. Davies, P. B. Jones et al., "Randomised controlled trials of conventional antipsychotic versus new atypical drugs, and new atypical drugs versus clozapine, in people with schizophrenia responding poorly to, or intolerant of, current drug treatment," Health Technology Assessment, vol. 10, no. 17, 2006.

[39] L. R. Derogatis, "The Derogatis Interview for Sexual Functioning (DISF/DISF-SR): an introductory report," Journal of Sex and Marital Therapy, vol. 23, no. 4, pp. 291-304, 1997.

[40] D. W. Heinrichs, T. E. Hanlon, and W. T. Carpenter, "The quality of life scale: an instrument for rating the schizophrenic deficit syndrome," Schizophrenia Bulletin, vol. 10, no. 3, pp. 388-398, 1984.

[41] A. F. Lehman, "Measures of quality of life among persons with severe and persistent mental disorders," Social Psychiatry and Psychiatric Epidemiology, vol. 31, no. 2, pp. 78-88, 1996.

[42] J. Cramer, R. Rosenheck, W. Xu, W. Henderson, J. Thomas, and D. Charney, "Detecting improvement in quality of life and symptomatology in schizophrenia," Schizophrenia Bulletin, vol. 27, pp. 227-234, 2001.

[43] D. Aizenberg, Z. Zemishlany, P. Dorfman-Etrog, and A. Weizman, "Sexual dysfunction in male schizophrenic patients," Journal of Clinical Psychiatry, vol. 56, no. 4, pp. 137-141, 1995.

[44] D. Novick, J. M. Haro, E. Perrin, D. Suarez, and J. M. Texeira, "Tolerability of outpatient antipsychotic treatment: 36-month results from the European Schizophrenia Outpatient Health Outcomes (SOHO) study," European Neuropsychopharmacology, vol. 19, no. 8, pp. 542-550, 2009.

[45] R. Gourevitch, S. Abbadi, and J. D. Guelfi, "Quality of life in schizophrenics with and without the deficit syndrome," European Psychiatry, vol. 19, no. 3, pp. 172-174, 2004. 


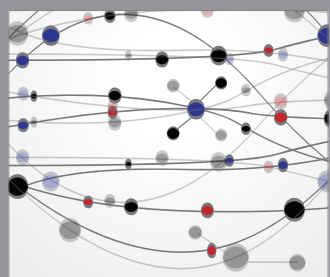

The Scientific World Journal
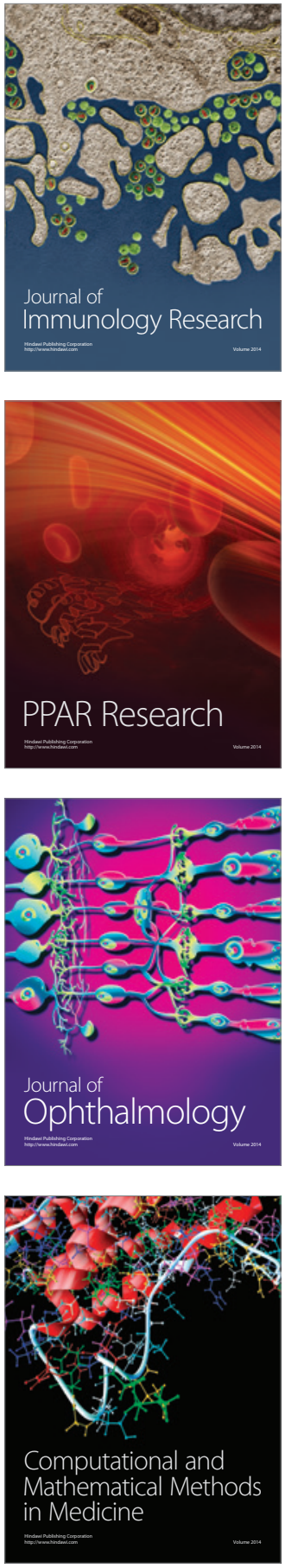



Gastroenterology

Research and Practice
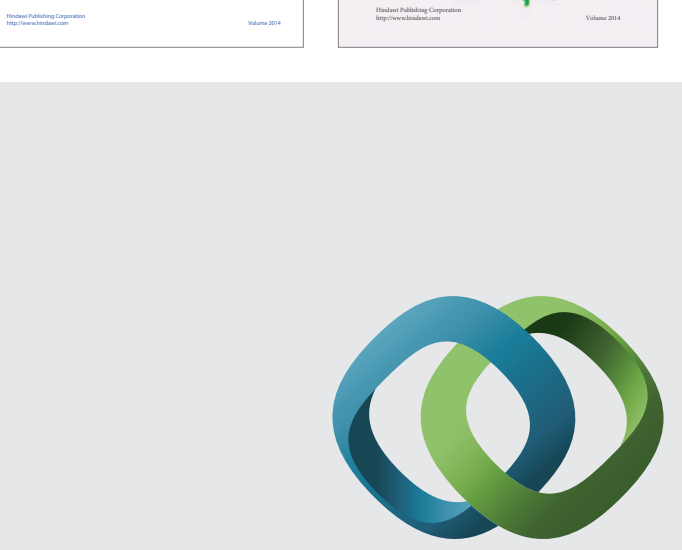

\section{Hindawi}

Submit your manuscripts at

http://www.hindawi.com
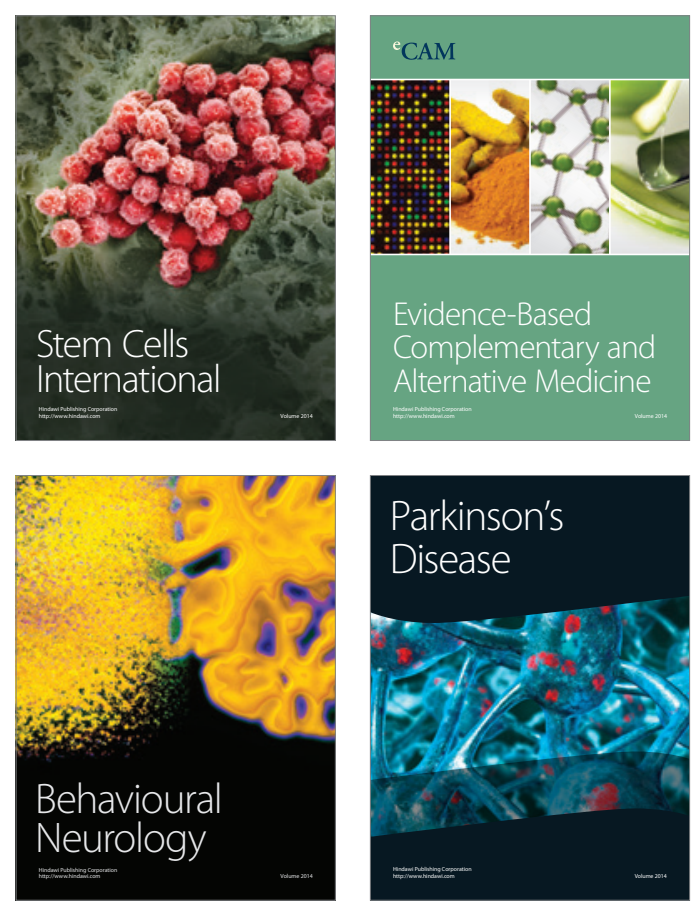

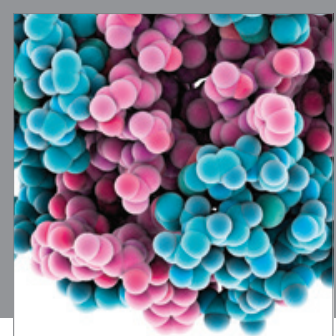

Journal of
Diabetes Research

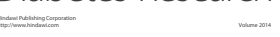



Disease Markers
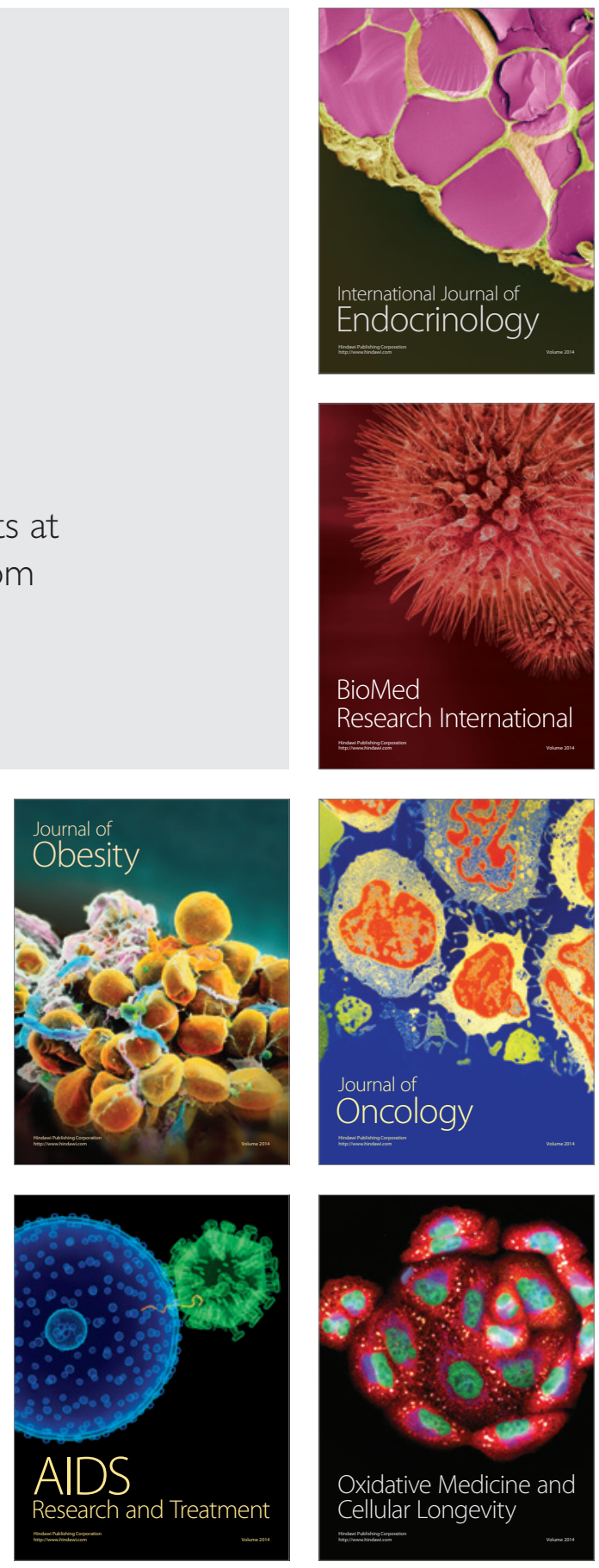